\title{
STUDY ON BALLISTOCARDIOGRAM ACQUISITION IN A MOVING WHEELCHAIR WITH EMBEDDED SENSORS
}

\author{
Eduardo Pinheiro ${ }^{1)}$, Octavian Postolache ${ }^{2)}$, Pedro Girão ${ }^{1)}$ \\ 1) Instituto de Telecomunicações and Instituto Superior Técnico/UTL, Av. Rovisco Pais, 1, 1049-001 Lisbon, Portugal \\ ( $₫$ eduardo.pinheiro@lx.it.pt, +351 218418 454,p.girao@lx.it.pt) \\ 2) Instituto de Telecomunicações IT-IUL/Instituto Universitário de Lisboa and Instituto Politécnico de Setúbal, Campus do IPS, Estefanilha \\ 2910-761 Setúbal, Portugal (opostolache@lx.it.pt)
}

\begin{abstract}
Embedding cardiac system sensing devices in wheelchairs is both necessary and attractive. Elders, diabetics, or stroke victims are a substantial group needing permanent cardiac monitoring, without restriction of their already limited mobility. A set of sensing devices was embedded in a wheelchair to monitor the user without his awareness and intervention. A dual-wavelength reflection photoplethysmogram (PPG), and a ballistocardiogram (BCG) based on MEMS accelerometers and on electromechanical film sensors are output by the hardware. Tests were conduced on twenty one subjects, for an immobility scenario. Additional recordings were made for helped propulsion over a tiled floor course, with good results in keeping track of acceleration BCG and PPG. A treadmill was also used for tests, providing a smooth floor and constant speed and inclination. The PPG and acceleration BCG could be continuously monitored in all the tests. The developed system proves to be a good solution to monitor cardiac activity of wheelchair users even during motion.
\end{abstract}

Keywords: cardiac continuous monitoring, photoplethysmogram, ballistocardiogram, electrocardiogram, accelerometry, treadmill, unobtrusive instrumentation, wheelchairs.

(C) 2012 Polish Academy of Sciences. All rights reserved

\section{Introduction}

Mobile solutions for patient cardiac monitoring are viewed with growing interest [1-2]. The gradual aging and the prevalence of cardiac pathologies in modern societies lead to an increase of the number of subjects requiring continuous monitoring of cardiovascular parameters. However, most non-invasive methods are obtrusive and inappropriate for monitoring during long time periods. Improvements on current implementations are frequently reported, with wireless and wearable devices promising to achieve ubiquity [1].

Elders and subjects with reduced mobility benefit from home care. This approach is also more economical, and relieves hospital staff from routine exams [3]. Embedding sensors to create an intelligent monitoring environment is a profitable manner of monitoring numerous subjects, without having to individually distribute a wearable device [4-5]. This profit arises from the unobtrusiveness of such monitoring devices, key factors on subjects' acceptance and satisfaction, as well as in diminishing the white coat effect [6].

Many wheelchair users, stroke victims in recovery, elders, and diabetics need permanent monitoring of their cardiovascular status [7]. Hence, embedding cardiac system sensing devices in wheelchairs is both necessary and attractive. Furthermore, given the lack of limitations to the subjects' movements, it is interesting to apply these devices, for instance, to monitor the elders' state without constraining their autonomy [8].

To realize unconstrained monitoring tasks, electrophysiological signals are of much interest. The acquisition of the electrocardiogram (ECG), photoplethysmogram (PPG), and 
ballistocardiogram (BCG) from the wheelchair user provides knowledge on both the electrical stimulus to the myocardium and on the heart's response [9]. Heart response is of utmost interest, as it indicates contractility maladies or debilitation, which the ECG does not portray. The amplitude of the BCG waves is an accurate and early indicator of life expectancy and of vulnerability to cardiovascular diseases [10]. Hence its acquisition is very significant.

Developments undertook by our group on unobtrusive vital signal acquisition have culminated in a very robust hardware setup. The goal of this paper is to present a measurement system embedded in a wheelchair, monitoring the user's cardiac activity in a set of different scenarios. Cardiac activity estimation, by means of PPG and acceleration BCG, is proved possible in the following cases: stopped wheelchair; helped propulsion on curvy course over a ceramic tiled floor; in an exercise treadmill at any reasonable speed and inclination. Validation tests involved over twenty volunteers of different nationality, age, gender, and anthropometric conditions, part of them also participating in the movement tests.

This paper is organized as follows: Section 2 presents a synthesis of the current solutions for ballistocardiographic sensing and processing. Section 3 describes the sensing device. Section 4 characterizes the volunteers, and discusses the results of the validation tests taken in three different scenarios. The paper is finalized with the conclusions of Section 5 and a reference list.

\section{State of the art of unobtrusive sensing solutions of cardiac activity}

The BCG registers the body's vibrations due to the cardiac and respiratory activity [11]. The recording of the forces produced during the cardiac cycle is approached by embedding force transducers in objects. Beds [12-13], chairs [14-15], wheelchairs [16-17], bathroom scales [18], or pillows [19-20] are possibilities with confirmed feasibility.

Electromechanical film sensors were at the origin of the rediscovery of ballistocardiography [21]. Other types of sensors have also been applied to gather the pulsate ballistic-like signals, from load cells [15] and air pressure sensors [22], to strain gauges [18] or FMCW radar technology [4]. According to the measurement principle, the ballistocardiogram can be called a displacement ballistocardiogram or acceleration ballistocardiogram [23].

The renewed interest in this signal is also due to its depiction of the heart's biomechanical status, Fig. 1. This signal has a set of W-shaped waves, the systolic waves [24], which are due to ejection and blood passage in the aorta region, having a strong relation with the volume of blood ejected by the heart [11]. The evolution amplitude over time of the BCG systolic waves' amplitude is related to the cardiac force decay. It was shown decades ago that the cardiac forces depicted in the BCG are accurate and early indicators of life expectancy, and exactly predict the susceptibility to future cardiac maladies [10]. Long-term recordings made with present day devices allow the study of heart rate variability, as well as the synchronization with ECG, and PPG [14], [25].

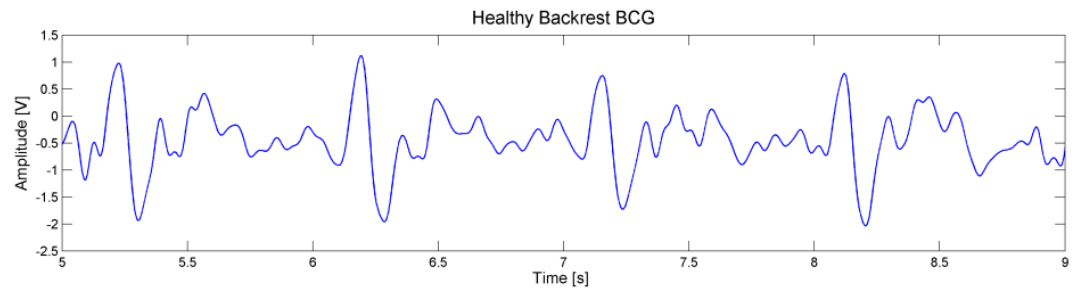

Fig. 1. Typical healthy BCG signal. Healthy subject with a $300 \times 290 \mathrm{~mm}^{2}$ film sensor embedded in a wheelchair's backrest. 
BCG sensing devices suffer greatly from movement artifacts, Fig. 2, and a great deal of research is being made on digital signal processing - from adaptive filters [18], to fusion of complementary information by accelerometry [16] or electromyography [26], as well as wavelet analysis [16], [27], and empirical mode decomposition [28] - to reduce such artifacts.
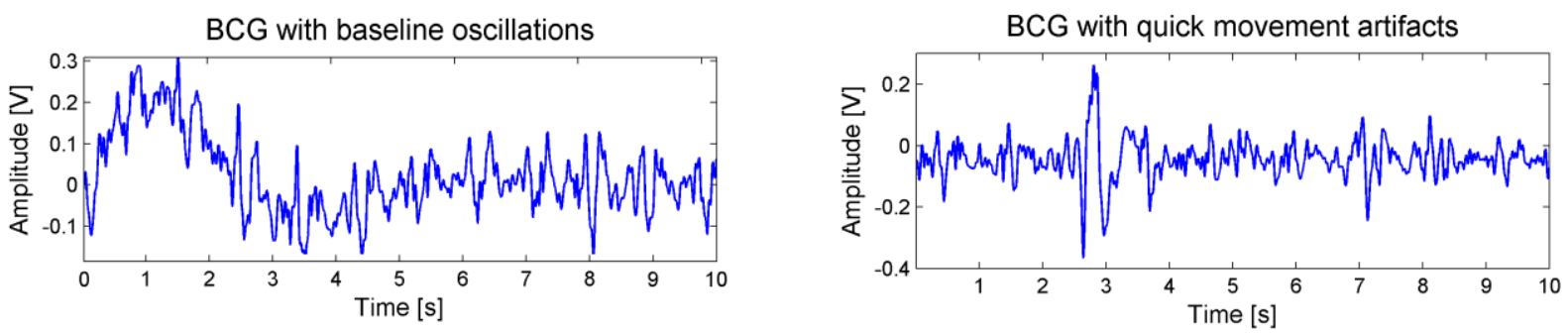

Fig. 2. Ballistocardiograms contaminated with noise due to subject movement, obtained with a $400 \mathrm{x} 300 \mathrm{~mm}^{2}$ film sensor. Slow baseline oscillations (left) and short but strong movements (right).

\section{Unobtrusive sensing system}

Given its justified interest, a wheelchair-based system to monitor a patient's cardiovascular condition was developed. It incorporates reflection photoplethysmography sensing in both armrests, and MEMS accelerometers and piezoelectric film sensors embedded in the backrest and in the seat. All signal-conditioning circuitry was installed in the wheelchair. This monitoring setup is totally unobtrusive, and the subject is not restrained in its daily use of the wheelchair. Fig. 3 presents a photograph of the wheelchair; one MEMS accelerometer and an EMFi sensor were placed over the seat for better visualization. For scale, a one-euro coin was also put over the $300 \times 290 \mathrm{~mm}^{2}$ film sensor. Both armrests have two pairs of light emitters and sensors to acquire the user photoplethysmogram.

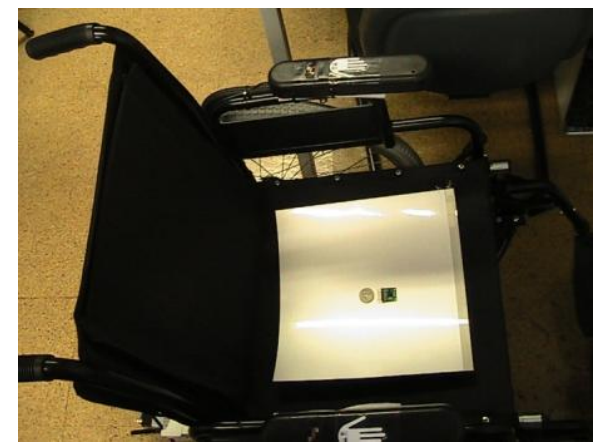

Fig. 3. Wheelchair implementing unobtrusive monitoring of user's functions. In the armrests the devices for PPG acquisition, and over the seat one accelerometer, a film sensor, and a one euro coin for scale.

The displacement BCG is monitored by two piezoelectric film sensing devices (EMFi) type EMFIT L-3030 embedded in the wheelchair's backrest and seat, beneath the polyester cover but over the lining. These sensors output a small charge variation with a typical sensitivity of $25 \mathrm{pC} / \mathrm{N}$. Their interior is compound of a thin porous polypropylene film structure, with several layers separated by air voids; when the exerted pressure changes, the voids change their shape, and charge movement is produced. Analogue signal conditioning circuitry amplifies and filters the sensors' output, with a low-pass cut-off frequency of $20 \mathrm{~Hz}$, which is a common value in BCG implementations [29].

The acceleration BCG is monitored by two MEMS accelerometers (Sparkfun ADXL335) placed behind the EMFi sensor. Their small size gives great importance to the choice of position. The option taken was the placement of the backrest sensor centered in the bottom, to minimize the effects of cervical and thoracic movements, and the seat sensor centered and 
about $3 \mathrm{~cm}$ from the back limit, to ensure proximity to the trunk support, thus mitigating the effects of leg movement or lateral leaning. The sensor outputs analogue voltages representing each axis' acceleration with a sensitivity of $1 \mathrm{~V} / \mathrm{G}$. Such implementation has been used to compensate artifacts [16], and may also be used to detect the user's transfer from or into the wheelchair.

The reflection PPG is obtained at two different wavelengths by a set of sensors and emitters embedded in both the wheelchair's armrests. Each armrest has a red pair, working at $645 \mathrm{~nm}$, composed of a LED (Multicomp MC20463) and a photodiode (Taos TSL250R), and an infrared pair, working at $940 \mathrm{~nm}$, composed of an emitter (Fairchild QEE113) and a photodiode (Taos TSL262R). The armrest implementation is depicted in Fig. 4.
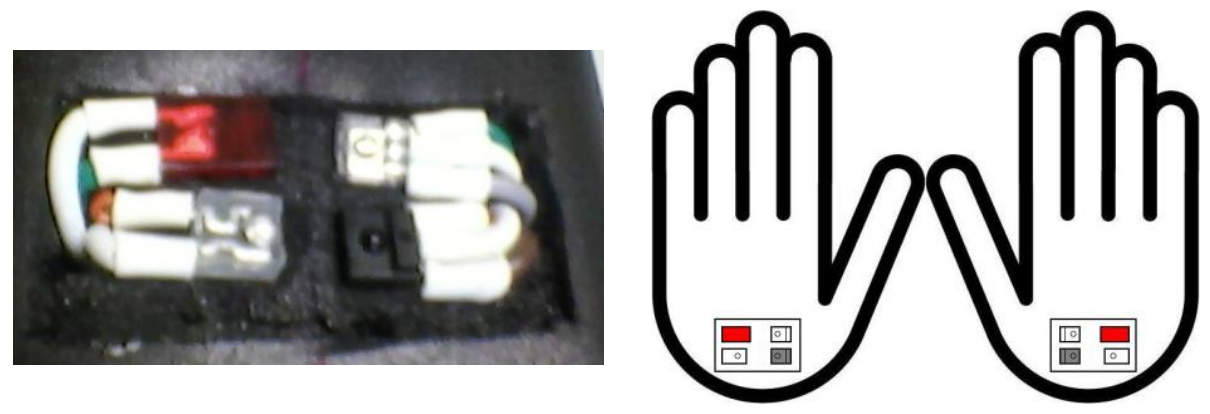

Fig. 4. Photo disclosing the optoelectronic devices placement on the left armrest (left), and intended placement of the hands over the armrests (right). The two emitters are on the same side, and in direct opposition to the sensor of the respective wavelength.

The red sensor maximum irradiance responsivity (top right in the photo of Fig. 4) is of 1.68 $\mathrm{V} /\left(\mu \mathrm{Wcm}^{-2}\right)$ at $645 \mathrm{~nm}$, while for the infrared sensor it is of $9.1 \mathrm{mV} /\left(\mu \mathrm{Wcm}^{-2}\right)$ at $940 \mathrm{~nm}$. The infrared emitter (bottom left in the photo of Fig. 4) radiant intensity is between 3 and 12 $\mathrm{mW} / \mathrm{sr}$, while the red emitter has a typical luminous intensity of 25 mcd.

Analogue signal conditioning circuitry multiplexes the light emission to minimize mutual interference. The limit in the commutation frequency is imposed by the rise/fall time of the red sensor, $160 \mu \mathrm{s}$. The photodiodes have their DC component removed by a $0.16 \mathrm{~Hz}$ filter, followed by an amplification stage, approximately leveling the amplitudes, and finally a filtering stage defines a common low-pass cutoff frequency of $20 \mathrm{~Hz}$.

To validate the sensing system a Medlab P-OX100 was used. This commercial equipment outputs three analogue voltages, the electrocardiogram (ECG) waveform, the instantaneous heart rate, and the oxygen saturation of the subject. These outputs are computed by means of the addition of a three-lead ECG and a closed finger PPG sensor. These three reference signals are also acquired by our system, so that synchronously recorded calibration data is available. A special-purpose plug is easily accessed from the wheelchair's rear, so that the equipment may be connected at any time.

The total amount of channels to record is 15: the 3 reference equipment outputs, plus the system outputs: four PPGs, two BCGs, and two tri-axial accelerations. The data is digitalized, and sent via WiFi to a laptop by means of a data acquisition board (National Instruments 9205), sampling the analogue inputs at $1 \mathrm{kHz}$, with 16 bits of resolution, plugged to a compatible wireless module (NI WLS-9163).

The signals sensed by the described hardware setup are exemplified in Fig. 5. The signals of one of the volunteers were normalized and shifted to their positions in the graph. In the first graph the ballistic signals are displayed. Besides the displacement BCGs, obtained through the electromechanical film sensors, the $z z$ axis accelerations are shown. The signals corresponding to the backrest and seat acceleration BCG are taken from this axis, while the other two are omitted. In the second graph the red and infrared reflection photoplethysmograms are displayed. 

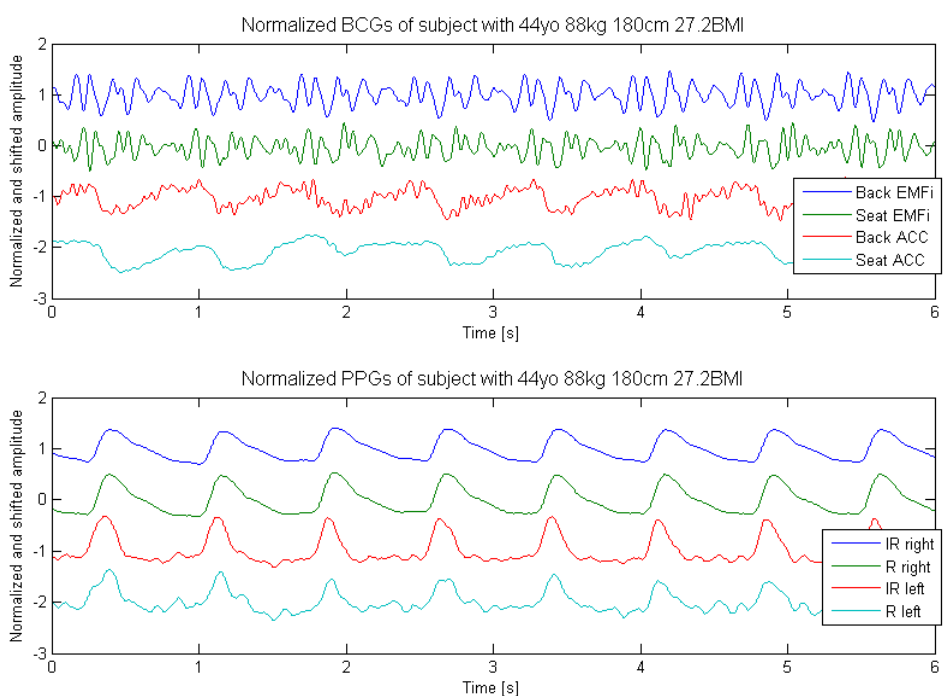

Fig. 5. All the physiological signals gathered by the system implemented in the wheelchair, simultaneously acquired for one of the volunteers. In the top, 4 BCGs, displacement, and acceleration, acquired in backrest and seat. In the bottom, 4 PPGs, red and infrared, acquired in both hands.

\section{Comparison Tests}

A total of 21 volunteers tested the system. Their characterization is provided in Fig. 6. Their age was $28.0 \pm 10.3$ years old (average \pm standard deviation), the weight was $71.4 \pm$ $10.8 \mathrm{~kg}$, and their height $1.74 \pm 0.09 \mathrm{~m}$. Seven subjects had a body to mass index (BMI) in the overweight (25-30) region, and the average BMI was 23.4.

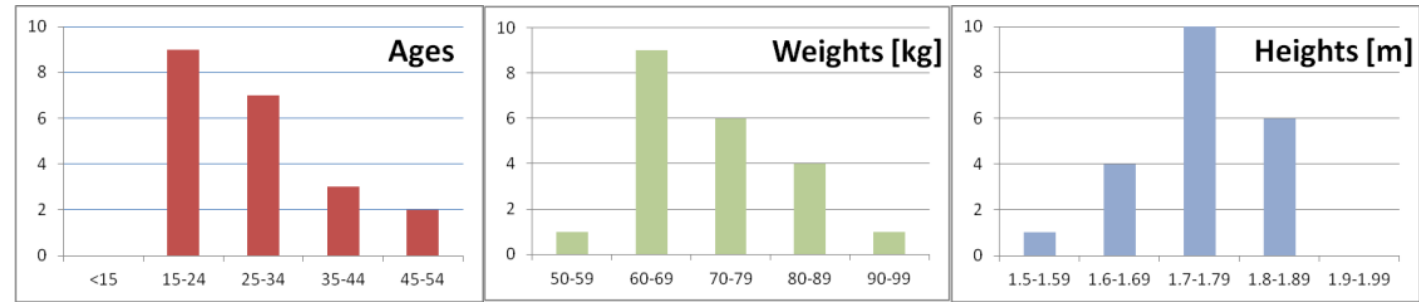

Fig. 6. Volunteers distributed by age (left), weight (middle), and height (right).

The 21 volunteers tested the sensing capabilities of the system with the wheelchair stopped. The acquisition was performed during 5 minutes, and the subject was still, but was allowed to talk normally. Eleven of the volunteers were then propelled on a predefined course during 3 minutes. The occupied wheelchair was pushed over an irregularly tiled floor, on a course with several $90^{\circ}$ turns and two $180^{\circ}$ turns. Treadmill recordings with variable inclination and speed were also made for one subject, 5 minutes were recorded in 17 different combinations to validate the device in a more controlled and repeatable environment.

\subsection{Unmoving wheelchair}

The subjects were seated on the wheelchair, and the finger PPG and 3-lead ECG of Medlab P-OX100 were connected. During 5 minutes the wheelchair did not move, and the subjects were told to be still, while allowed to talk normally.

In this immobility scenario, all the sensors worked properly, as exemplified in previous Fig. 5, and very clear quality signals were obtained. The subjects with most extreme characteristics have a portion of their signals presented in Fig. 7. To improve Fig. 7 
readability, the acceleration BCG was multiplied by 100 (equivalent to $100 \mathrm{~V} / \mathrm{g}$ ), and some signals were shifted upwards or downwards. The maximum static and dynamic loads are far from being reached, and the signal corresponding to the reflected light in the fingers is much superior to the ambient light transmitted through the hands. All the signals are well demarcated in all the subjects, confirming the validity of the developed hardware.

The BMI and weight variation are factors of direct influence in the BCG, as the sensitivity of the transducers depends on the static load. The subject height was taken as an indicator of different seating postures and the different positions of the force sensors regarding the heart. The subject age is important, given the known relation between cardiac force and age [10], and therefore different waveforms are expected. None of these elements is of special interest to the PPG signal, which, contrarily to the BCG, has a very repeatable pattern.
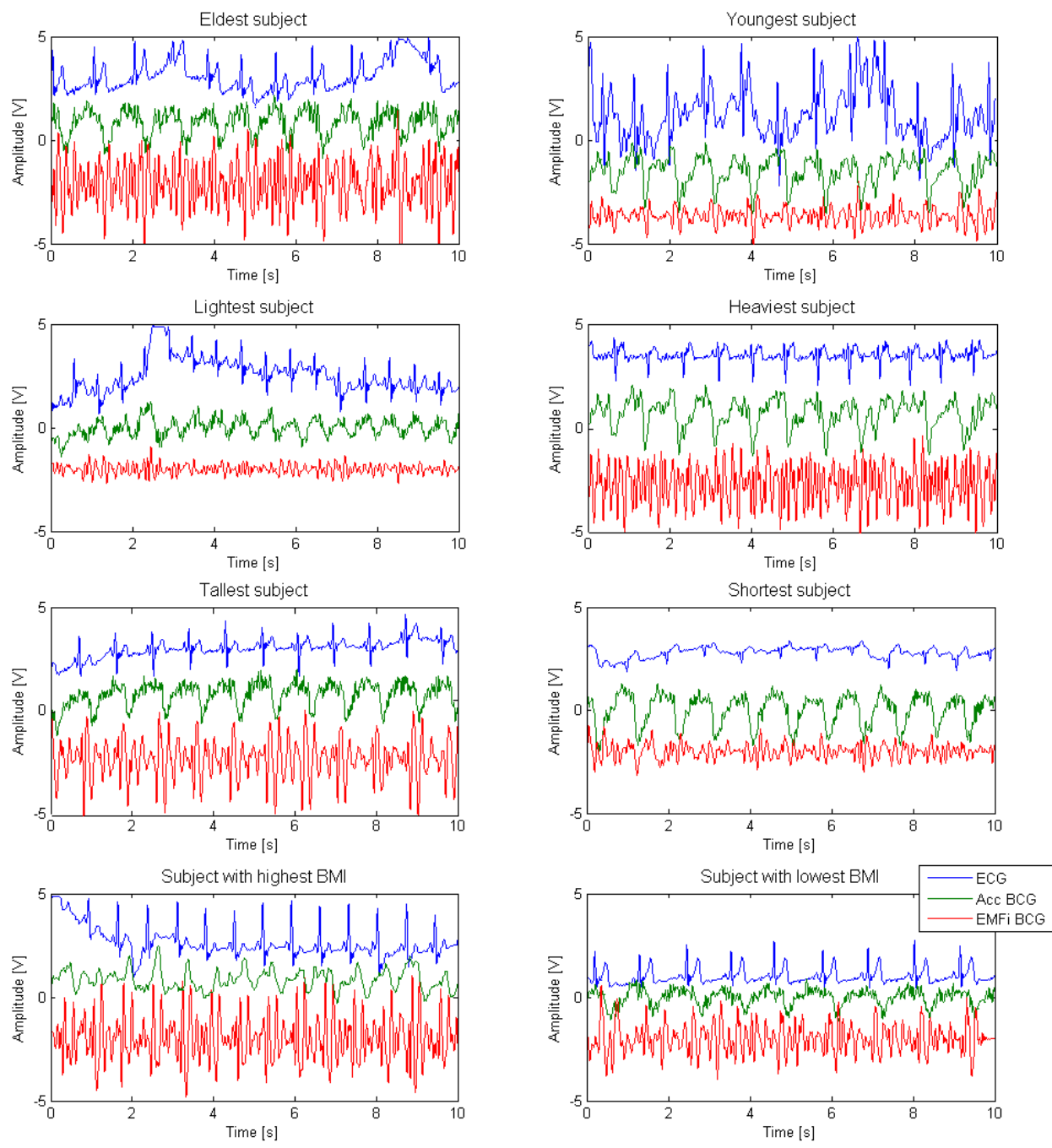

Fig. 7. Signals acquired from 8 subjects, with the wheelchair stopped. Each graph shows ECG from Medlab P-

OX100 (blue, upper position within graph) and seat BCG from zz axis of the accelerometer (green, middle position) and EMFi (red, bottom position). The eldest subject is 48 years old, the youngest 18. The lightest has $55 \mathrm{~kg}$, the heaviest $90 \mathrm{~kg}$. The tallest has $1.88 \mathrm{~m}$, the shortest $1.63 \mathrm{~m}$. The highest BMI is 29.4 , the lowest 19.4 .

The sensitivity of the EMFi sensor depends on the static load applied. However, it was not verified a modification of the BCG amplitude in direct relation with the subject weight, or BMI. Exemplifying, the second row of signals in Fig. 7 presents a weight difference of $35 \mathrm{~kg}$, whereas the last presents a weight difference of $25 \mathrm{~kg}$, and no deterministic amplitude relation exists. So, the blood ejection force is, together with the seating posture (defining the force propagation), the dominating factor on the amplitude. 
Regarding the morphology, the acceleration curves obtained (green signals in Fig. 7) present a single peak. The acceleration BCG does not depict the minor ballistic waves, related to the diastole, nor it presents the $\mathrm{W}$ shape usual in the systolic waves, therefore it has limited sensitivity comparing with the displacement BCG. Nonetheless, it has a constant and predictable shape, whereas the EMFi signal is very different from subject to subject, due to its better sensitivity.

\subsection{Tiled floor motion}

When moving in a tiled floor, undesired vibrations are registered together with the BCG. In Fig. 8 and Fig. 9 these vibrations are shown to be in the 4 to $10 \mathrm{~Hz}$ range. Fig. 8 presents the signals in the time domain, whereas Fig. 9 presents their spectral composition. Their cause is most probably structural vibrations stimulated by wheelchair movement together with tile bumping. As the EMFi sensor has a large area, not entirely compressed by the body, flexural vibrations of the marginal portions of the sensor may be considered as well.

Seat ballistocardiograms from six subjects, other than the ones already shown in the previous section, are displayed in Fig. 8. Fig. 9 presents the respective Fast Fourier Transforms to allow inspection of the frequency components present. To improve Fig. 8 readability the acceleration BCG was multiplied by 100, and the signals were shifted upwards and downwards. In Fig. 9 the amplitudes were normalized to allow comparison.
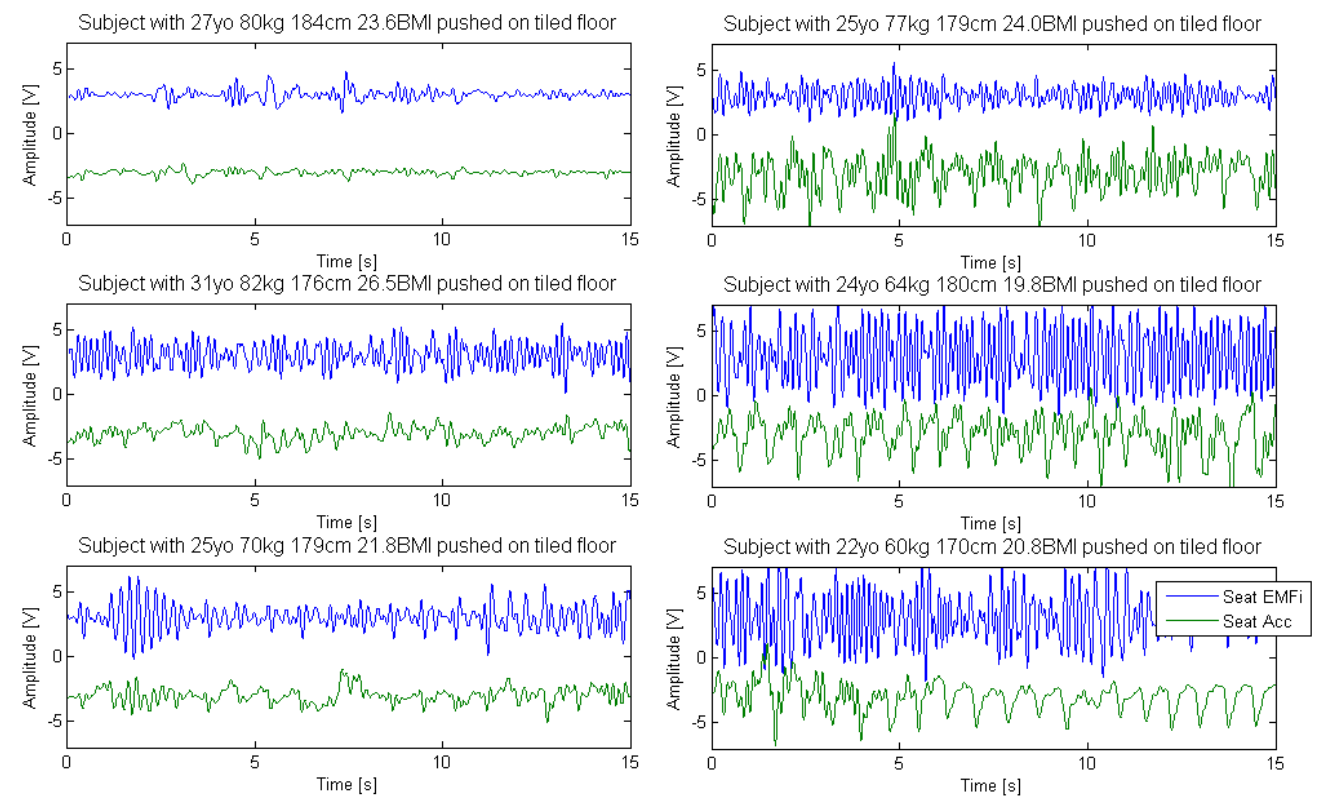

Fig. 8. Signals acquired from 6 subjects, pushed on the wheelchair over the tiled floor shown. The seat EMFi signal (blue upper position) and the seat accelerometer zz axis (green lower position) are shown.

As it is shown in Fig. 8, the cardiac portion of the acceleration BCG amplitude does not depend on the subject weight. Although the lighter subjects have clear acceleration curves, the $3^{\text {rd }}$ lightest has a very low-amplitude signal (left bottom), contrarily to what happens to a $7 \mathrm{~kg}$ heavier subject (top right). Seating position, or body fat distribution, are more probable explanations for the curves behaviour.

Frequently, the cardiac information present in film sensors is impossible to determine due to the superposition of high-amplitude motion-induced noise. This is verified both in backrest or seat implementations, at almost any speed, whereas the accelerometer is much more insensitive to wheelchair motion. However, the acceleration sensors may also fail, whenever the person is not sufficiently supported in his back, or bouncing laterally, but in ordinary use 
of the wheelchair it is possible to obtain the BCG signal. In fact, the accelerometer proves to be a reliable source to acquire ballistocardiographic data, as exemplified in the results shown above, especially when installed in the seat.

To analyze the superposition between the cardiac signal and vibration noise, the FFT was applied. From Fig. 5 it is understandable that a clean acceleration BCG signal does not have significant harmonic content. In fact, the major peak of the spectral amplitude is related to the heart rate frequency, and the acceleration BCG signal is fully contained in the 0-3 Hz band. The structural vibrations, visible for instance in the top right graph of Fig. 9, appear in the 4-6 $\mathrm{Hz}$ band, thus without much direct interference over the region of interest.

Analyzing Fig. 9 it is seen that the seat accelerometer $\mathrm{zz}$ axis (green) has a more modest component in the 3-18 Hz interval than the seat EMFi signal (blue). In fact, a clean cardiac signal obtained from the accelerometer has all the information in the $0-3 \mathrm{~Hz}$ range, which can be verified in middle and bottom right figures. Comparing with the homologue graphs of Fig. 8 it is seen that the signals with clearer cardiac peaks have the most reduced component in the 3-18 Hz interval.
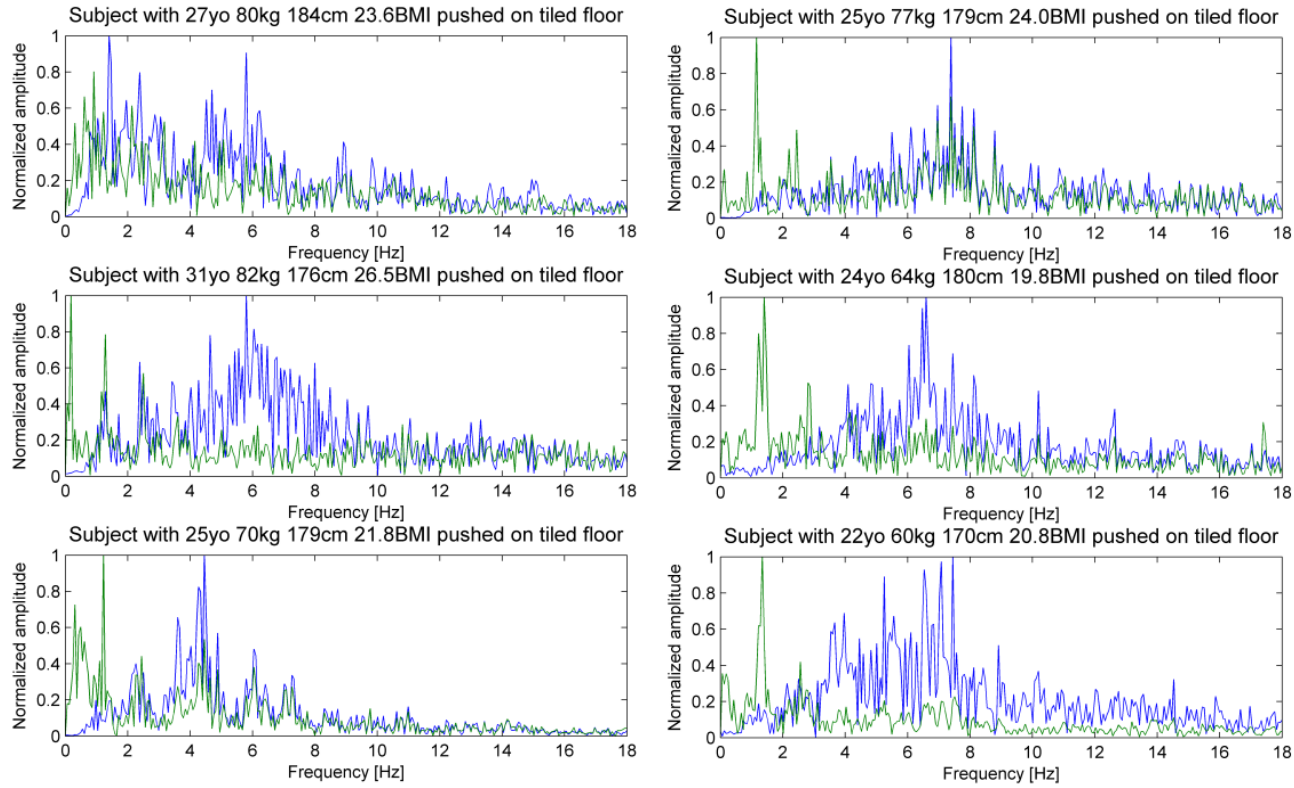

Fig. 9. Normalized amplitude spectrum of the homologue signals presented in Fig. 8, acquired from 6 subjects, pushed on the wheelchair over a tiled floor. Seat accelerometer zz axis (green) and EMFi (blue) are displayed.

The BCG signal produced by the film sensor is more affected by spurious vibrations generated by the wheelchair movement. For instance, the signal of Fig. 1 has its content within $2-7 \mathrm{~Hz}$, with a single major peak at $4 \mathrm{~Hz}$. Such distribution indicates that the waveform has much more harmonic content, and minor waves, than the acceleration BCG signal, and multiples of the heart rate appear. However, only the bottom left graph of Fig. 9 is near such distribution. This is coherent with the fact that only the homologue graph of previous Fig. 8 presents a portion of a clear cardiac signal. No other spectrum has a prominent peak around 4 Hz. Further, the peaks near 6 or $7 \mathrm{~Hz}$ are only related to structural vibrations, as no cardiac signal is visible in the other graphs of Fig. 8. Thus, it is evident that the spectrum is completely corrupted, and the peak in 6 or $7 \mathrm{~Hz}$ is by no means related with cardiac activity.

Simultaneous recording of the PPG was done and provided the signals represented in Fig. 10. In the middle left graph, a pair of sensors was not being covered by the volunteer's hand, so the reflection signals have an erratic behavior, or saturate. Signals corresponding to hand movements are displayed in the other graphs of the left column. 

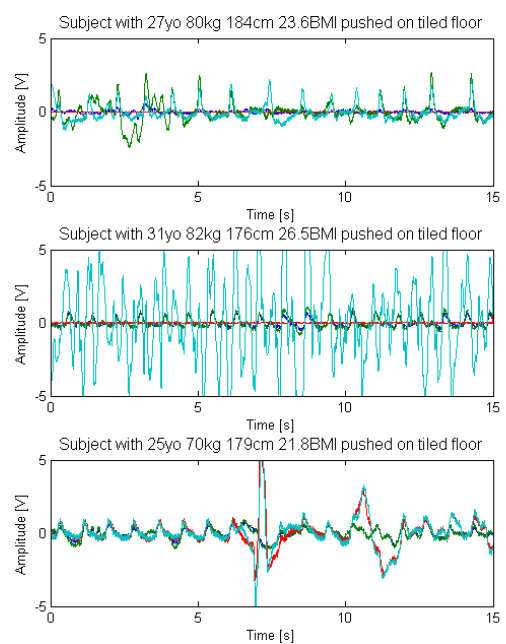
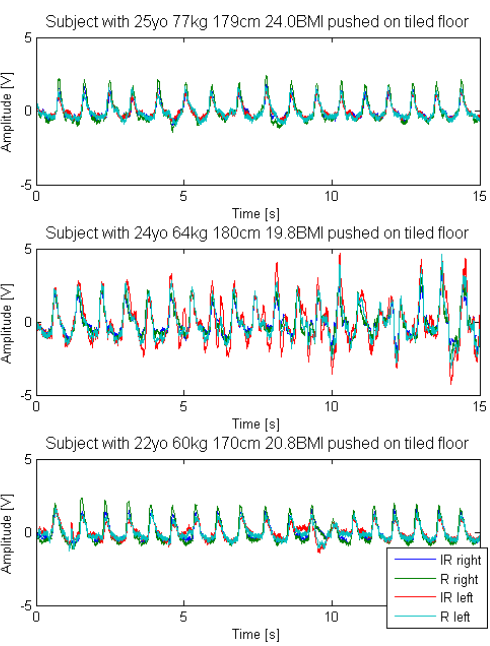

Fig. 10. PPG signals, acquired simultaneously with the homologue signals presented in Fig. 8, from 6 subjects pushed on the wheelchair over tiled floor. The right hand infrared (blue), right hand red (green), left hand infrared (red) and red (light blue) signals are shown.

Analyzing Fig. 10 it is seen that when the subject does not move his hands, and both are in a similar position, the 4 signals acquired are almost identical (right column). If one hand is out of the armrest, sensors saturate or provide very high amplitude outputs (middle left). When there is hand motion relatively to the armrest, artifacts are generated. Generally, it was confirmed that when the hands do not move over the PPG transducers, the vibrations to which the wheelchair is subject are not important.

\subsection{Treadmill tests}

A test session was made with one volunteer in a professional gym treadmill with variable speed and inclination, Pulse Fitness 260FT. Several speeds and inclinations were experimented, and for each combination a 5-minute recording was done. Fig. 11 depicts a tensecond period of some recordings, with inclinations of $0 \%$ and $4.4 \%$, and with speeds of 1,3 , 5 and $7 \mathrm{~km} / \mathrm{h}$. The ECG, the signal from the electromechanical film and accelerometer embedded in the seat, and the backrest acceleration are shown. The acceleration BCGs are multiplied by 100 , and the EMFi by $1 / 5$ to improve legibility.

It is clear from the example that the seat acceleration BCG (green) is more stable and immune to high frequency vibratory noise than the backrest acceleration BCG (red). When speed increases, the backrest sensor is corrupted by wheelchair vibration at frequencies comparable to the seat film sensor, whereas the seat accelerometer is not interfered. Therefore, the seat accelerometer is the best device to continuously monitor the ballistocardiogram even when the wheelchair is moving.

When not subject to accelerations, the EMFi provides a signal definition which the accelerometers do not have sensitivity to match. However, only in the 1 and $3 \mathrm{~km} / \mathrm{h}$ flat floor recordings a signal with fundamental frequency similar to the cardiac beat can be seen, and even so highly interfered. In flat floor movement, any of the accelerometers is preferable to the electromechanical film, which was the same result obtained from the tiled floor tests. Accelerometers are a better solution for general movement scenarios due to their noise immunity, but higher sensitivity would be desirable to improve the ballistic wave definition.

At higher speeds it is visible that the EMFi signal is periodic, around 300 to $500 \mathrm{~ms}$. But, as the ECG confirms, this periodicity has no connection with cardiac activity. Hence it may be related with the mechanical vibrations of the whole structure. Frequency estimation algorithms may need to be specifically tuned to avoid misclassifying tachycardia. Another important fact is that the spurious vibrations emerging from movement on the smooth 
treadmill mat are similar to the ones on the tiled floor. Therefore, the cause of such vibrations is motion itself, and not tile bumping.
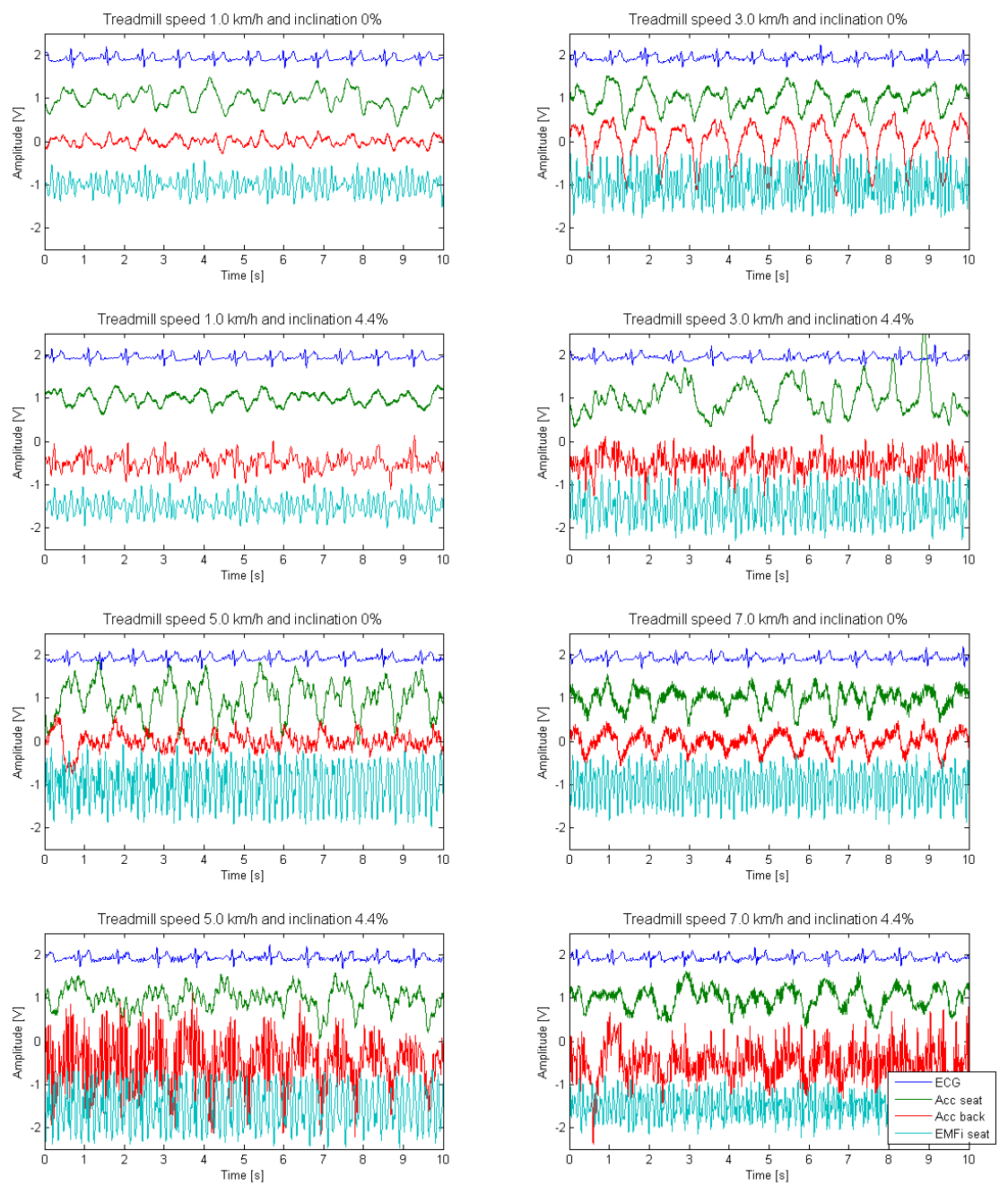

Fig. 11. Signals acquired from 1 subject at 8 different motion regimes with the wheelchair in the Pulse Fitness 260 FT treadmill. Each graph shows ECG from Medlab P-OX100 (blue, upper position within graph) and seat BCG from zz axis of accelerometer (green, upper middle position), backrest BCG from zz axis of accelerometer (red, lower middle position) and seat EMFi (light blue, lower position).

It is important to mention that although the treadmill provided a smooth pavement, it was never possible to obtain the BCG from the film sensor. Such happened at all the programmed speeds and inclinations. Therefore, the problems verified in previous Section 4.2 were not due to tile bumping, but to the structural vibrations of the wheelchair when moving. However, positive results on continuous monitoring with acceleration BCG, and PPG were again verified for inclinations up to $4.4 \%$ and speeds up to $7 \mathrm{~km} / \mathrm{h}$.

\section{Conclusions}

An unobtrusive cardiac sensing unit was developed and implemented in a wheelchair, acquiring signals of its occupant and transmitting them wirelessly. This hardware platform acquires dual wavelength reflection PPG in armrests, displacement BCG and acceleration from the backrest and the seat. This embedded sensing solution for cardiac activity monitoring is attractive. It is able to gather the photoplethysmogram, displacement and acceleration ballistocardiogram signals, and transmit them wirelessly to a laptop. 
Twenty one healthy subjects with diverse anthropometric characteristics, age (between 18 and 48), height (1.50 and $1.88 \mathrm{~m}$ ), weight (55 and $90 \mathrm{~kg}$ ), and BMI (19.3 and 28.3) tested this immobility scenario, and all of them were able to be monitored continuously.

Additional recordings were made for helped propulsion over a tiled floor course, with good results in keeping a continuous track of acceleration BCG and PPG. The displacement BCG, obtained through an electromechanical film sensor, depicts the several events of the cardiac cycle, while the acceleration BCG presents only the ejection peak. However, during motion the acquired EMFi BCG waveforms cannot provide heart rate information. In the acceleration $\mathrm{BCG}$ the heart rate is detectable for all the subjects, in spite of the floor induced vibrations, and $90^{\circ}$ curves. Although not being perfect for speeds up to $7 \mathrm{~km} / \mathrm{h}$ and inclinations up $4.4 \%$, and on reasonable floor types, the sensing methodology is able to surpass the movement scenario conditions.

A treadmill was also used for tests with one subject. It provided a smooth pavement and programmable speed and inclinations. The PPG and acceleration BCG could be continuously monitored in all the tests, for inclinations up to $4.4 \%$ and speeds up to $7 \mathrm{~km} / \mathrm{h}$. The waveforms acquired are of good quality, and heart rate and the saturation of peripheral oxygen may be estimated. The system developed proves to be a good solution to monitor cardiac activity of wheelchair users even during motion in demanding conditions.

Future work on this system will relate with embedding a capacitive ECG sensor to increase the amount of cardiac data available, as well as to provide a more familiar signal to the evaluation staff. The addition of data processing capabilities at the wheelchair level will also be evaluated. Instead of transmitting the waveforms acquired, it is intended to estimate immediately the user's homeostasis parameters. The addition of computation power locally will ultimately enable automatic communication with the wheelchair user, and in case of failure to respond, to an emergency contact.

\section{Acknowledgements}

Instituto de Telecomunicações (IT) and Fundação para a Ciência e Tecnologia (grant SFRH/BD/46772/2008 and project RIPD/APD/109639/2009) kindly supported this work.

\section{References}

[1] Kumar, S., Kambhatla, K., Hu, F., Lifson, M., Xiao, Y. (2008). Ubiquitous computing for remote cardiac patient monitoring: a survey. International Journal of Telemedicine and Applications, (1), 1-19.

[2] Postolache, O., Girão, P.S., Pinheiro, E.C., Postolache, G. (2010). Unobtrusive and non-invasive sensing solutions for on-line physiological parameters monitoring. Lay-Ekuakille, A., Mukhopadhyay, S. (eds.). Wearable and Autonomous Biomed. Devices and Systems for Smart Environment, 75, Springer, 277-314.

[3] Myers, S., Grant, R., Lugn, N., Holbert, B., Kvedar J. (2006). Impact of home-based monitoring on the care of patients with congestive heart failure. Home Health Care Management \& Practice, 18(6), 444-451.

[4] Postolache, O. Girão, P., Pinheiro, E., Pereira, J., Madeira, R., Postolache, G., Mendes, J. (2011). Multiusage of microwave doppler radar in pervasive healthcare systems for elderly. In Proc. of the IEEE Instrumentation and Measurement Technology Conference, Hangzhou, China, 30-34.

[5] Uenoyama, M., et al. (2006). Non-contact respiratory monitoring system using a ceiling-attached microwave antenna. Medical and Biological Engineering and Computing, 44(9), 835-840.

[6] Berntson, G., Cacioppo, J., Quigley, K., Fabro, V. (Jan. 1994). Autonomic space and psychophysiological response. Psychophysiology, 31(1), 44-61.

[7] Kaye, H., Kang, T., LaPlante, M. (2000). Mobility device use in the United States. National Institute on Disability and Rehabilitation Research, Washington, USA. 
[8] Chan, L., Zhang, J., Narayanan, M., Celler, B., Lovell, N. (2008). A health monitoring and evaluation system for assessing care needs of residents in aged-care facilities. In Proc. of the $6^{\text {th }}$ IASTED International Conference on Biomedical Engineering, Innsbruck, Austria, 414-418.

[9] Pinheiro, E.C., Postolache, O., Girão, P.S. (2010). Non-intrusive device for real-time circulatory system assessment with advanced signal processing capabilities. Measurement Science Review, 10(5), 166-175.

[10] Starr, I., Wood, F. (1961). Twenty-year studies with the ballistocardiograph: the relation between the amplitude of the first record of 'healthy' adults and eventual mortality and morbidity from heart disease. Circulation, 23(5), 714-732.

[11] Pinheiro, E., Postolache, O., Girão, P. (2010). Theory and developments in an unobtrusive cardiovascular system representation: ballistocardiography. The Open Biomedical Engineering Journal, 4, 201-216.

[12] Lim, Y., et al. (March 2011). Monitoring physiological signals using nonintrusive sensors installed in daily life equipment. Biomedical Engineering Letters, 1(1), 11-20.

[13] Mendez, M., Matteucci, M., Cerutti, S., Bianchi, A., Kortelainen, J. (2009). Automatic detection of sleep macrostructure based on bed sensors. In Proc. of the $31^{\text {st }}$ IEEE EMBC, Minneapolis, USA, 5555-5558.

[25] Pinheiro, E.C., Postolache, O., Girão, P.S. (2009). Pulse arrival time and ballistocardiogram application to blood pressure variability estimation. In Proc. of the IEEE Int. W. Med. Meas. Applic., Cetraro, 132-136.

[15] Su, J., Zhu, X., Zhang, X., Tang, J., Liu, L. (2009). Ballistocardiogram measurement system using three load-cell sensors platform in chair. In Proc. of the $2^{\text {nd }}$ Int. Conf. on Biomedical Engineering and Informatics, Tianjin, China, 1-4

[16] Postolache, O., Girão, P.S., Mendes, J., Pinheiro, E.C., Postolache, G. (2010). Physiological parameters measurement based on embedded sensors in a wheelchair and advanced signal processing. IEEE Trans. on Instrumentation and Measurement, 59(10), 2564-2574.

[17] Kim, J., Hong, J., Cho, M., Cha, E., Lee, T. (2007). Wireless biomedical signal monitoring device on wheelchair using noncontact electro-mechanical film sensor. In Proc. of the $29^{\text {th }}$ IEEE EMBC, Lyon, France, 574-577.

[18] Inan, O., Etemadi, M., Widrow, B., Kovacs, G.(2010). Adaptive cancellation of floor vibrations in standing ballistocardiogram measurements using a seismic sensor as a noise reference. IEEE Trans. on Biomedical Engineering, 57(3), 722-727.

[19] Chen, W., Zhu, X., Nemoto, T., Kanemitsu, Y., Kitamura, K., Yamakoshi, K. (Apr. 2005). Unconstrained detection of respiration rhythm and pulse rate with one under-pillow sensor during sleep. Medical and Biological Engineering and Computing, 43(2), 306-312.

[20] Zhu, X., et al. (2006). Real-time monitoring of respiration rhythm and pulse rate during sleep. IEEE Trans. on Biomedical Engineering, 53(12), 2553-2563.

[21] Koivistoinen, T., Junnila, S., Varri, A., Koobi, T. (2004). A new method for measuring the ballistocardiogram using EMFi sensors in a normal chair. In Proc. of the $26^{\text {th }}$ IEEE EMBC, San Francisco, USA, 1, 2026-2029.

[22] Chee, Y., Han, J., Youn, J., Park, K. (2005). Air mattress sensor system with balancing tube for unconstrained measurement of respiration and heart beat movements. Physiological Measur., 26(4), 413.

[23] Scarborough, W., et al. (1956). Proposals for ballistocardiographic nomenclature and conventions: revised and extended: report of committee on ballistocardiographic terminology. Circulation, 14(3), 435-450.

[24] Pollock, P. (May 1957). Ballistocardiography: a clinical review. Canad. Medi. Assoc. J., 76(9), $778-783$.

[25] Alametsä, J., Viik, J., Palomäki, A. (2008). Arterial elasticity measurements with ankle pulse width velocity and ballistocardiography. In Proc. of the $4^{\text {th }}$ Eur. Conf. of the IFMBE, Antwerp, 22, 1636-1641.

[26] Inan, O., Etemadi, M., Wiard, R., Giovangrandi, L., Kovacs, G. (2008). Evaluating the foot electromyogram signal as a noise reference for a bathroom scale ballistocardiogram recorder. In Proc. of the $21^{\text {st }}$ IEEE Int. Symp. on Computer-Based Medical Systems, Jyväskylä, Finland, 70-74.

[27] Jin, J., Wang, X., Li, S., Wu, Y. (2009). A novel heart rate detection algorithm in ballistocardiogram based on wavelet transform. In Proc. of the $2^{\text {nd }}$ Int. W. Known. Disc. Data Min., Porto, Portugal, 76-79.

[28] Pinheiro, E., Postolache, O., Girão, P. (2012). Empirical mode decomposition and principal component analysis implementation in processing non-invasive cardiovascular signals. Measurement, 45(2), 175-181.

[29] Junnila, S., Akhbardeh, A., Barna, L., Defee, I., Varri, A. (2006). A wireless ballistocardiographic chair. In Proc. $28^{\text {th }}$ IEEE EMBC, New York, USA, 5932-5935. 